This is an Author's Original Manuscript of an article submitted for consideration in the International Journal of Psychology, copyright Taylor \& Francis http://www.tandfonline.com/doi/abs/10.1080/00207594.2012.677539

\title{
Cognitive control in belief-laden reasoning during conclusion processing: An ERP study
}

Junlong Luo ${ }^{1}$, Xiumin $\mathrm{Du}^{2,3}$, Edward J. N. Stupple ${ }^{4}$, Xiao Xiao ${ }^{1}$, Lei Jia ${ }^{1}$, Qinglin Zhang ${ }^{1,5}$

${ }^{1}$ Department of Psychology, Southwest University, Chongqing 400715, China;

${ }^{2}$ Institute of Psychology, Chinese Academy of Sciences, Beijing 100101, China;

${ }^{3}$ Graduate School of Chinese Academy of Sciences, Beijing 100049, China;

${ }^{4}$ Centre for Psychological Research, University of Derby, UK

${ }^{5}$ Key Laboratory of Personality and Cognition, Ministry of Education, Southwest

University, Chongqing 400715, China

\author{
Correspondence to: $\quad$ Qinglin Zhang \\ School of psychology \\ Southwest University, \\ Beibei, Chongqing 400715 China \\ and \\ Edward J. N. Stupple \\ Lecturer Centre for Psychological Research, \\ University of Derby, UK \\ E-mail address: luo831023@163.com; E.J.N.Stupple@derby.ac.uk \\ Junlong Luo and Xiumin Du contributed equally to this work
}


This research was supported by the National Key Discipline of Basic Psychology in Southwest University (NSKD08002) and the National Natural Science Foundation of China (30970892, 31170983)

Abstract: Belief-bias is the tendency to accept conclusions that are compatible with existing beliefs more frequently than those that contradict beliefs. It is one of the most replicated behavioral findings in the reasoning literature. Recently, neuroimaging studies using functional magnetic resonance imaging (fMRI) and event-related potentials (ERPs) have provided a new perspective and have demonstrated neural correlates of belief bias that have been viewed as supportive of dual process theories of belief bias. However, fMRI studies have tended to focus on conclusion processing, while ERPs studies have been concerned with the processing of premises. The electrophysiological correlates of cognitive control were studied among 12 subjects using high density ERPs. The analysis was focused on the conclusion presentation phase and was limited to normatively sanctioned responses to valid-believable and valid-unbelievable problems. Results showed that when participants gave normatively sanctioned responses to problems where belief and logic conflicted, a more positive ERP deflection was elicited than for normatively sanctioned responses to non-conflict problems. This was observed from -400 to $-200 \mathrm{~ms}$ prior to the correct response being given. The positive component is argued to be analogous to the late positive component (LPC) involved in cognitive control processes. This is consistent with the inhibition of empirically anomalous information when conclusions are unbelievable. 
These data are important in elucidating the neural correlates of belief-bias by providing evidence for electrophysiological correlates of conflict resolution during conclusion processing. Moreover, they are supportive of dual process theories of belief bias that propose conflict detection and resolution processes as central to the explanation of belief bias.

Keywords: Belief-bias effect; Cognitive control; Dual Processing; Event-related potentials (ERPs); Late positive component (LPC)

\section{Introduction}

The belief bias effect in syllogistic reasoning is the tendency of participants to accept more believable conclusions than unbelievable conclusions; the bias is more pronounced for invalid problems but is also observed for valid problems, such that the conclusion-endorsement rates display an interaction between logical status and the believability of the problems (e.g., Evans, 2003; Klauer, Musch, \& Naumer, 2000). In the traditional belief-bias paradigm there are two types of syllogisms: conflict problems, in which the logical conclusion is inconsistent with one's beliefs (valid-unbelievable and invalid-believable) and non-conflict problems in which the logical conclusion is consistent with one's beliefs (valid-believable and invalid-unbelievable). Most accounts of belief bias attempt to explain the observed interaction in conclusion endorsement rates with dual-process theories. These accounts propose two types of cognitive process: Type 1 processing entails rapid belief-driven heuristics, whereas, Type 2 processing entails slower analytic responding (e.g., Evans, 2003, 2007). These differing processes come into conflict 
when their outcomes differ. Studies have demonstrated robust effects of response patterns, response times and confidence ratings within the belief bias paradigm (e.g., Evans, Barston, \& Pollard, 1983; Prowse Turner \& Thompson, 2009; Stupple \& Ball, 2008) which are well explained by these dual process accounts. For example, dual process theories have gained support for the prediction of increased response times for conflict problems relative to non-conflict problems due to the cognitively demanding process of belief inhibition (e.g., Stupple \& Ball, 2008). Furthermore, Evans \& Curtis-Holmes (2005) demonstrated increased belief bias for speeded responses which is consistent with limited time reducing the opportunity for Type 2 processing. Moreover, Stupple, Ball, Evans \& Kamal-Smith (in press) have shown that cognitive ability moderates the influence of belief bias, such that, the most able participants on belief bias tasks devote additional processing time to the most demanding conflict problems. These example studies are illustrative of the general literature that is, for the most part, consistent with dual process theories that place conflict detection and resolution as central to the explanation of belief bias.

Furthermore, neuroscientific methods, such as functional near infrared spectroscopy (fNIRS), functional magnetic resonance imaging (fMRI) and event-related potentials (ERPs) have demonstrated the value of utilising new measures to examine the belief bias effect (Goel \& Dolan, 2003; Luo, Yang, Du, \& Zhang, 2011; Luo, Yuan, Qiu, Zhang, Zhong, \& Huai, 2008; Tsujii, Masuda, Akiyama, \& Watanabe, 2010; Tsujii \& Watanabe, 2010). This accumulating neuroscientific evidence has generally shown support for dual process theories (see e.g., De Neys, 
Moyens, \& Vansteenwegen, 2010) and has added weight to the argument that belief bias is difficult to explain with a unitary cognitive process (but see, Dube, Rotello and Heit, 2010 for a challenge to dual process theory from a single-process perspective).

Much of this neuroscientific evidence centers on cognitive control - the ability to guide thought and action in accordance with internal intentions (Cohen, Botvinick, \& Carter, 2000), which plays an important role in situations requiring planning, decision making, conflict resolution, and error correction (Posner \& DiGirolamo, 1998). Dual process accounts of belief bias posit cognitive control mechanisms such as belief inhibition, analytic inhibition and conflict monitoring (e.g., Evans, 2007; Stupple et al., in press) as central to explaining the phenomenon. For example, normative responses to valid-unbelievable problems require both the detection of the conflict between belief and logic, and the inhibition of the belief to avoid rejection of the valid conclusion. Invalid-believable problems require even greater cognitive control as they also require the inhibition of an analytic bias towards searching for models that are consistent with belief. This evidence from fMRI and fNIRS studies demonstrates that the inhibition of belief-bias, is associated with increased activation of the right lateral prefrontal cortex (PFC), (Goel \& Dolan, 2003; Tsujii, et al., 2010; Tsujii \& Watanabe, 2010).

Though the fMRI and fNIRS technique can provide accurate information about the location of the brain areas involved, these measures are limited in their capacity to inform us about the timing of activations. Event-related potentials (ERPs) provide a means to evaluate timing of cognitive processes prior to a response, with this 
technique, recordings are made of the electrical activity of the brain that are time-locked to the presentation of an external stimulus. ERP data allow for a precise examination of the time course of activation for different stages of reasoning. This approach has been successfully applied to both the reasoning domain and more specifically for conflict resolution. For example, studies (Luo et al., 2011; Luo et al., 2008) have found some ERP components associated with the belief-bias effect. However, since these studies only examined the temporal resolution of premise processing instead of the processing of conclusions, it remained unclear what happens to the ERP effects in the conclusion presentation phase after the presentation of the premises. This is important because, when ERP measures are timelocked to premise presentation, it is only possible to obtain an incomplete picture of the process because belief-logic conflicts can only emerge once the conclusion has been presented. Furthermore, there is evidence that belief biased reasoning is conclusion driven (e.g., Morley, Evans \& Handley, 2004), and as such the examination of ERPs timelocked to conclusion presentation would seem to be essential. In addition, Bonnefond and Van der Henst (2009) highlighted the fact that most fMRI studies have focused on conclusion processing and, in the interests of the comparability of studies, it is clear that the examination of conclusion processing is an important next step.

In the present study, the neural basis of inhibitory processing (before participants make a correct response on a logical conclusion) in belief-laden syllogistic reasoning was investigated using high-density (64 channels) ERP recording system. Participants were asked to perform a syllogistic reasoning task, involving two types of belief-bias 
problems: conflict problems (with valid-unbelievable conclusions) and non -conflict problems (with valid-believable conclusions), and were required to make a normatively sanctioned logical response after conclusion presentation. Thus participants needed to inhibit their beliefs for the conflict condition but not in the non-conflict condition.

Previous studies (Luo et al., 2011; Luo et al., 2008) have provided evidence for electrophysiological correlates of belief-bias effect during premise processing. For example, a greater negativity was associated with the detection of conflict between empirical beliefs and logical rules in conditional reasoning (Luo et al., 2011). Similarly, it has been postulated that an increased N2 in conditional reasoning is related to perceptual conflict (Bonnefond \& Van der Henst, 2009; Prado, Kaliuzhna, Cheylus, \& Noveck, 2008). Conversely, a greater positivity was found in syllogistic reasoning when participants are faced with beliefs that are inconsistent with logical rules (Luo et al., 2008). The authors' interpretation of this finding was that the greater positivity (or LPC) may reflect the mental inhibition of conflict. Similarly, in the previous literature, LPC (which also is called the "P3", see Polich, 2007) is an index of neural inhibition (Donchin, 1981; Polich, 2007). The available research, however, appears to be inconsistent, which may be due to the different reasoning paradigms (syllogistic reasoning versus conditional reasoning) in these studies. In view of using syllogistic reasoning (consistent with Luo et al., 2008) in the present study, we hypothesized that the LPC) would be involved in inhibiting the belief-bias effect for the conflict problems. By recording and analyzing high-density ERPs elicited by 
making a logical response under different conditions, the ERP data will allow for a more precise examination of the time course of conflict resolution during conclusion processing.

\section{Materials and methods}

\subsection{Participants}

As paid volunteers, 12 undergraduate students (6 women, 6 men) aged 21-24 years (mean age, 22.8 years) participated in the study. All participants were right-handed, and with no reported neurological disorders. This study was approved by the local Ethics Committee, and all participants signed an informed consent form for the experiment.

\subsection{Materials}

\section{INSERT TABLE1 ABOUT HERE}

In order avoid the influence of belief of the premises, we selected an atypical material to ensure the premises are empirically true. The specific format of reasoning is as follows: All $A$ are $B$ (major premise). All $C$ are $B$ (minor premise). Therefore, the relation between $A$ and $C$ is indefinite (valid conclusion) / Therefore, all $A$ are $B$ (invalid conclusion). Two task conditions (conflict and non-conflict, see Table 1) were examined when this reasoning format was filled by empirically true premises. In order to exclude the influence of differing decisions (acceptance and rejection), only trials with correct response (i.e., acceptance) on valid conclusions of conflict/non-conflict conditions were taken into ERP analysis. To ensure that there were enough trials available for each subject and condition in a reasonable time frame, 
the ratio of correct to incorrect conclusions (i.e., conflict and non-conflict) was 3:1. Furthermore, we selected other formats of reasoning with concrete content as additional materials, for example: (1) All $A$ are B; All B are $C$; Therefore, all $A$ are $C$ (valid conclusion) / the relation between $A$ and $C$ is indefinite (invalid conclusion). (2) All $B$ are $C ;$ All $A$ are $B ;$ Therefore, all $A$ are $C$ (valid conclusion) / the relation between $A$ and $C$ is indefinite (invalid conclusion). (3) All A are B; All A are $C$; Therefore, some $B$ are $C$ (valid conclusion) / the relation between $B$ and $C$ is indefinite (invalid conclusion). The three reasoning formats were filled by empirically true premises too. Thus, the additional materials entail conflict and non-conflict problems, but the inhibition of belief-bias in these problem types would not be investigated. These additional materials were used to ensure the effective inference during premise onset, in case participants judged the validity of the conclusions without inference. The ratio of correct to incorrect additional conclusions was 1:3, which is a balance for whole ratio. And the number of conflict trials, non-conflict trials, and additional reasoning trials were 80,80 , and 160, respectively. A baseline condition was not devised in this study since the premises are all empirically true. Thus, it is difficult to find an ideal baseline in neural activations that are associated with inhibiting belief-bias during conclusion processing. The presented words and sentences are familiar by people in their daily life (e.g., women, flower and animal). The length of each conclusion was eight to thirteen Chinese characters, and the characters were high-frequency words. The focus of the analysis in the present study upon the valid problems is driven by the desire to maximize the number of 
participants who respond correctly. Stupple et al. (in press) demonstrated that only the most able sub-set of participants consistently provide the normatively sanctioned response to invalid-believable problems, as such it was prudent to exclude these problems from the analysis.

\subsection{Procedure}

\section{INSERT FIGURE 1 ABOUT HERE}

The overall experiment was divided into two parts: a training phase and a test phase. The training phase consisted of at least 3 blocks in order to train participants to inhibit their belief-bias. Each block (i.e., conflict condition, non-conflict condition and additional condition) contained one condition (10 trails). Participants were given feedback after each trial, and those whose accuracy rate in every condition was greater than $80 \%$ were allowed to begin the experiment. The formal test stage was composed of five blocks, with each block incorporating 64 trials (i.e., 16 conflict trials, 16 non-conflict trials, and 32 additional reasoning trials). The stimuli presented in the test blocks were randomized across the three categories of conflict condition, non-conflict condition and additional condition.

Subjects were seated in a semi-dark room facing a monitor placed $60 \mathrm{~cm}$ away from the eye. Horizontal and vertical visual angles were below $5^{\circ}$. Each reasoning item was presented in the following way as shown in Figure 1. Each trial was initiated by a "+" in the center of screen for $300 \mathrm{~ms}$. Next, the major premise (six to eight Chinese characters at a visual angle of $1.93^{\circ} \times 0.81^{\circ}$ ) and the minor premise (six to eight Chinese characters) were shown for $5,000 \mathrm{~ms}$, during which subjects were 
instructed to draw a logical conclusion based on the major and minor premises. Subsequently, the blank screen that lasted for $200-400 \mathrm{~ms}$ was followed by the presentation of a conclusion for $2500 \mathrm{~ms}$. Finally, subjects were asked to judge whether or not the conclusion was valid or not by pressing different keys (' 1 ' if the conclusion is correct; ' 2 ' if the conclusion is incorrect) as quickly and as accurately as possible on a keypad. In addition, they were instructed to keep their eyes fixated on the center of the monitor during the experiment to avoid ocular artifacts.

\subsection{Electrophysiological recording and analysis}

Brain electrical activity was recorded at 64 scalp sites using tin electrodes mounted in an elastic cap (Brain Product, Brain Products GmbH, Gilching, Germany), with the reference on the left and right mastoids. The vertical electrooculogram (EOG) was recorded with electrodes placed above and below the left eye. All interelectrode impedance was maintained below $5 \mathrm{k} \Omega$. The EEG and EOG were amplified using a 0.05-100 $\mathrm{Hz}$ bandpass and continuously sampled at $500 \mathrm{~Hz} / \mathrm{channel}$ for off-line analysis. Eye movement artifacts (blinks and eye movements) were rejected offline. Trials with EOG artifacts (mean EOG voltage exceeding $\pm 80 \mu \mathrm{V}$ ) and those contaminated with artifacts due to amplifier clipping, bursts of electromyographic (EMG) activity, or peak-to-peak deflection exceeding $\pm 80 \mu \mathrm{V}$ were excluded from averaging.

The ERP waveforms were response-locked to the onset of the conclusions. Response-locked ERPs were calculated for a 900ms epoch, extending from 700ms before the response until 200ms (baseline) after the response. The reason for the 
window of analysis is $700 \mathrm{~ms}$ is that the shortest RTs to the conclusions are $716 \pm 121$ ms (non-conflict conditions). Only trials with correct response on valid conclusions of conflict/non-conflict conditions were taken into ERP analysis. On the basis of the grand averaged ERPs and topographical map (see Fig. 2), the following 33 electrode positions (including anterior, central, parietal, posterior and occipital sites) were chosen for statistical analysis: Fz, F1, F2, F3, F4, FCz, FC1, FC2, FC3, FC4, Cz, C1, C2, C3, C4, CPz, CP1, CP2, CP3, CP4, Pz, P1, P2, P3, P4, POz, PO3, PO4, PO5, PO6, Oz, O1 and O2. Mean amplitudes in the time windows of -700 to $-600 \mathrm{~ms},-600$ to $-500 \mathrm{~ms},-500$ to $-400 \mathrm{~ms},-400$ to $-300 \mathrm{~ms},-300$ to $-200 \mathrm{~ms}$ and -200 to $-100 \mathrm{~ms}$ were analyzed using a two-way repeated-measures analyses of variance (ANOVA). The ANOVA factors were task type (two levels: conflict condition and non-conflict condition), and electrode site. In case of violations of sphericity assumptions, degrees of freedom were corrected by means of the Greenhouse-Geisser method.

\section{Results}

A threshold alpha level of .05 was set for all analyses.

\subsection{Behavioral data}

The average number of conflict and non-conflict trials that participants responded to correctly was $53 \pm 7(87.8 \pm 11.6 \%)$ and $58 \pm 3(96.5 \pm 5.1 \%)$, respectively. Repeated-measures ANOVA on the accuracy rates showed that the effect of task type was significant $\left(F(1,11)=10.172, p=.009, \eta_{\mathrm{p}}{ }^{2}=.48\right)$. Mean reaction times $(\mathrm{RTs})$ for conflict conditions and non-conflict conditions were $747 \pm 149 \mathrm{~ms}$ and $716 \pm 121 \mathrm{~ms}$, respectively. The repeated-measures ANOVA on the reaction times (RTs) showed 
that the effect of task type was approaching the threshold significance level in the predicted direction $\left(F(1,11)=4.015, p=.070, \eta_{\mathrm{p}}^{2}=.27\right)$.

\subsection{Electrophysiological scalp data}

\section{INSERT FIGURE 2 ABOUT HERE}

Based on the grand average waveforms and difference wave map (Fig. 2), we found that correct response for conflict trials elicited a more positive ERP deflection than non-conflict trials from -400 to $-200 \mathrm{~ms}$ before the response.

The two factor repeated measures ANOVA showed main effects of task type in the time windows of -400 to $-300 \mathrm{~ms}$ and -300 to $-200 \mathrm{~ms}$ separately, $F(1,11)$ $=7.692, p=.018, \eta_{\mathrm{p}}{ }^{2}=.71$ and $F(1,11)=11.279, p=.006, \eta_{\mathrm{p}}{ }^{2}=.86$. The significant difference between conflict and non-conflict trials was specified for the interval of -400 to $-200 \mathrm{~ms}$. Neither the main effect of the electrode site nor the interaction of the task type and the electrode site was significant in any time window.

\section{Discussion}

In the present study, the participants were required to evaluate a logical conclusion based on empirically true premises and make a logical judgment after conclusion presentation. The electrophysiological data suggested that conflict problems elicited a more positive ERP deflection than the non-conflict problems at -400 to $-200 \mathrm{~ms}$ prior the correct response on valid conclusions. Behavioral data showed that mean accuracy rate was higher for the non-conflict condition than for the conflict condition, thus replicating the belief-bias effect to some extent using these atypical materials. However, the RTs data did not show statistically significant support for the difference 
between conflict and non-conflict conditions.

The finding on RTs runs counter to evidence presented by Stupple and Ball (2008) indicating that RTs for conflict condition are reliably slower than for non-conflict condition. This finding is explicable for two reasons: first, smaller sample sizes in neuroimaging studies are common and the trend shown in the data suggests that a larger sample would be likely to show statistically significant support for the reaction time effect. Moreover, the present study focused upon the less demanding valid-believable and valid-unbelievable problems, whereas, Stupple \& Ball found the largest differences between invalid-believable problems and non-conflict problems. As such these behavioural data are not inconsistent with the findings of Stupple and Ball or with theories that predict increased response times for conflict problems through an effortful process of belief inhibition.

In previous studies (e.g., Evans et al., 1983), where participants had not received logical training, the accuracy rate of logically correct answers for conflict condition was only $46 \%$. In contrast these accuracy data demonstrated a high logical accuracy in conflict condition, most likely due to logical training, however, this was necessary to obtain a sufficient sample of correct responses for an ERP analysis.

Premises were presented prior to conclusions and, it can be reasonably inferred that participants had begun to formulate an integrated model of the premises prior to the conclusion presentation phase. Previous evidence has shown conflict detection processing occurring during the premise processing (Luo et al., 2008; 2011) and the present study extends that finding to the conclusion processing phase. In order to 
resolve the conflict between belief and logic to endorse valid-unbelievable problems, participants needed to inhibit their beliefs, whereas this inhibition was absent during responses to non-conflict problems. Thus participants exerted greater cognitive effort in inhibiting the empirically anomalous information, to avoid a non-logical response.

As shown in Fig. 2, at the -400 to -200 ms time window, conflict conditions elicited an increased positivity, compared to non-conflict conditions, prior to the selection of the correct response. Together with the mean RTs, we can infer that the positive component peaks with a latency of $300-500 \mathrm{~ms}$ after the conclusion onset. Based on peak latency, the positive component is similar to the ERP component LPC (although it cannot definitively be described as LPC because the ERP analysis is time-locked to the response phase). The comparison of the positive component with LPC (or P3) is interesting because it is related to the inhibitive process. It has been widely accepted that $\mathrm{P} 3$ reflect neural inhibition of concomitant cognitive processes (Donchin, 1981; Polich, 2007). Previous research has generally found that more intense inhibitive processes evoke larger P3 amplitudes (Donkers \& van Boxtel, 2004; Pfefferbaum, Ford, Weller, \& Kopell, 1985). Therefore, we argue that the evident positive component elicited by conflict conditions may be attributed to a belief inhibition process when conclusions were empirically false. This finding is, therefore, of particular interest to dual process theorists as it shows that there are neural correlates of conflict resolution during conclusion processing in belief bias. These data are, moreover, inconsistent with single process theories (e.g., Dube et al. 2010) as they provide evidence consistent with cognitive control or belief inhibition which 
was observed only for the belief-logic conflict condition.

The present study demonstrated that a greater positive component was elicited by the conflict condition than the non-conflict condition between -400 and $-200 \mathrm{~ms}$ before correct response on logical conclusions, which, it is argued is related to the cognitive control of empirically false information in the conflict condition. Further study is needed to test and verify the LPC-like component in this paradigm and examine the contrast with previous ERP studies in this domain. Nonetheless, the presented data are parsimoniously explicable by dual process theorists (e.g., Evans, 2007), and are problematic for those theorists who advocate against dual process theories of reasoning (e.g., Keren \& Schul, 2009). 


\section{References}

Bonnefond, M., \& Van der Henst, J.B. (2009). What's behind an inference? An EEG study. Neuropsychologia, 47, 3125-3133.

Cohen, J. D., Botvinick, M., \& Carter, C. S. (2000). Anterior cingulate and prefrontal cortex: Who's in control? Nature Neuroscience, 3, 421-423.

De Neys, W., Moyens, E., \& Vansteenwegen, D. (2010). Feeling we're biased: Autonomic arousal and reasoning conflict. Cognitive, Affective, and Behavioral Neuroscience, 10, 208-216

Donchin, E. (1981). Surprise!... surprise? Psychophysiology, 18(5), 493-513.

Donkers, F. C. L., \& van Boxtel, G. J. M. (2004). The N2 in go/no-go tasks reflects conflict monitoring not response inhibition. Brain and Cognition, 56(2), 165-176.

Dube, C., Rotello, C. M., \& Heit, E. (2010). Assessing the Belief Bias Effect With ROCs: It's a Response Bias Effect. Psychological Review, 117(3); 831-863.

Evans, J.S.B.T. (2003). In two minds: dual process accounts of reasoning. Trends in Cognitive Sciences, 7, 454-459.

Evans, J. St. B. T. (2007). On the resolution of conflict in dual process theories of reasoning. Thinking \& Reasoning. 13:4, 321-339.

Evans, J.S.B.T., Barston, J. L., \& Pollard, P. (1983). On the conflict between logic and 
belief in syllogistic reasoning. Memory \& Cognition, 11, 295-306.

Evans, J. St. B. T., \& Curtis-Holmes, J. (2005). Rapid responding increases belief bias: Evidence for the dual-process theory of reasoning. Thinking \& Reasoning, 11, 382-389.

Goel, V., \& Dolan, R.J. (2003). Explaining modulation of reasoning by belief. Cognition, 87, 11-22.

Keren, G., \& Schul, Y. (2009) Two is not always better than one: A critical evaluation of two-system theories. Perspectives on Psychological Science, 4, 533-550

Klauer, K.C., Musch, J., \& Naumer, B. (2000). On belief bias in syllogistic reasoning. Psychological Review, 107, 852-884.

Luo, J. L., Yang, Q., Du, X. M., \& Zhang, Q. L. (2011). Neural correlates of belief-laden reasoning during premise processing: An ERP study. Neuropsychobiology, 63,112-118.

Luo, J. L., Yuan, J. J., Qiu, J., Zhang, Q. L., Zhong, J., \& Huai, Z. C. (2008). Neural correlates of the belief-bias effect in syllogistic reasoning: an event-related potential study. Neuroreport, 19, 1075-1079.

Morley, N. J., Evans, J. St. B. T., \& Handley, S. J. (2004). Belief bias and figural bias in syllogistic reasoning. Quarterly Journal of Experimental Psychology, 57A, 666-692.

Prado, J., Kaliuzhna, M., Cheylus, A., \& Noveck, I. A. (2008). Overcoming perceptual features in logical reasoning: An event-related potentials study. Neuropsychologia, 46(11), 2629-2637. 
Pfefferbaum, A., Ford, J. M., Weller, B. J., \& Kopell, B. S. (1985). ERPs to response production and inhibition. Electroencephalography and clinical neurophysiology, 60(5), 423-434.

Polich, J. (2007). Updating P300: an integrative theory of P3a and P3b. Clinical Neurophysiology, 118, 2128-2148.

Posner, M.I., \& DiGirolamo, G. J. (1998). In: R. Parasuraman (Eds.), The attentive brain, MIT Press, pp. 402-423.

Stupple, E. J. N., \& Ball, L. J. (2008). Belief-logic conflict resolution in syllogistic reasoning: Inspection-time evidence for a parallel process model. Thinking \& Reasoning, 14, 168-189.

Stupple, E. J. N., \& Ball, L. J., Evans, J. St. B. T., Kamal-Smith, E.N. (in press). When logic and belief collide: Individual differences in reasoning times support a selective processing model. Journal of Cognitive Psychology

Tsujii, T., Masuda, S., Akiyama, T., \& Watanabe, S. (2010). The role of inferior frontal cortex in belief-bias reasoning: An rTMS study. Neuropsychologia, 48(7), 2005-2008.

Tsujii, T., \& Watanabe, S. (2010). Neural correlates of belief-bias reasoning under time pressure: A near-infrared spectroscopy study. Neuroimage, 50, 1320-1326. 


\section{Figure and Table captions}

Fig. 1. Stimulus paradigm presentation

Fig. 2. Grand average ERPs at $\mathrm{Fz}, \mathrm{Cz}$, and $\mathrm{Pz}$ for correct response for conflict conditions, non-conflict conditions, and the difference wave (conflict condition minus non-conflict condition). Bottom right Topographical maps of the voltage amplitudes for the difference wave from -400 to $-340 \mathrm{~ms}$, -340 to $-280 \mathrm{~ms}$, and -280 to $-220 \mathrm{~ms}$

Table 1 Examples of task types with approximate literal translation. The premises for all tasks are empirically true 